\title{
Epidural hematoma treated by aspiration after transforaminal epidural steroid injection - A case report -
}

\author{
Go Eun Kim, Sung Jun Hong, Sang Soo Kang, Ho Joon Ki, \\ and Jae Hyun Park
}

Received October 27, 2020

Revised February 24, 2021

Accepted March 2, 2021

Department of Anesthesiology and Pain Medicine, Kangdong Sacred Heart Hospital, College of Medicine, Hallym University, Seoul, Korea

\section{Corresponding author}

Go Eun Kim, M.D.

Department of Anesthesiology and Pain Medicine, Kangdong Sacred

Heart Hospital, College of Medicine, Hallym University, 150 Seongan-ro, Gangdong-gu, Seoul 05355, Korea Tel: 82-2-2224-2659

Fax: 82-2-474-0956

Email: rhdnsn188@naver.com
Background: Spinal epidural hematoma is rare condition that can rapidly develop into severe neurologic deficits. The pathophysiology of this development remains unclear. There are several case reports of emergency hematoma evacuations after epidural steroid injection.

Case: We report on two patients who developed acute, large amounts of epidural hematoma without neurological deficits after transforaminal epidural steroid injection. After fluoroscopy guided aspiration for epidural hematoma was performed, neurological defects did not progress and the hematoma was shown to be absorbed on magnetic resonance imaging.

Conclusions: These reports are believed to be the first of treating epidural hematoma occurring after transforaminal epidural steroid injection through non-surgical hematoma aspiration. If large amounts of epidural hematoma are not causing neurological issues, it can be aspirated until it is absorbed.

Keywords: Epidural injection; Low back pain; Magnetic resonance imaging; Spinal epidural hematoma; Spinal stenosis.
Low back pain (LBP), which is the most common health problem resulting in pain and disability, is present in up to $70 \%$ of adults aged 60 years old or older and its incidence increases with age [1]. Many studies have shown that epidural steroid injection can be helpful for relief of radicular pain. Known serious complications after epidural anesthesia, such as epidural hematoma, abscesses, and arachnoiditis, are uncommon with a reported incidence of approximately $1 / 150,000$. However, the prevalence after transforaminal epidural steroid injection (TFESI) has not been studied [2]. We report on two patients who developed acute, severe back pain without neurological deficits after receiving TFESI due to large amounts of epidural hemato- ma. Fluoroscopy-guided aspiration for epidural hematoma was performed on these two patients, resulting in their back pain changing from severe to mild. Neurological defects did not progress and absorption of the hematoma was shown on magnetic resonance imaging (MRI) within two or three weeks. Physicians often decide to treat epidural hematoma through emergency surgery because patients suffer motor deficits and cauda equina syndrome. However, if large amounts of epidural hematoma are not causing neurological deficits, it can be aspirated until it is absorbed.

This is an Open Access article distributed under the terms of the Creative Commons Attribution Non-Commercial License (http://creativecommons.org/licenses/by-nc/4.0) which permits unrestricted non-commercial use, distribution, and reproduction in any medium, provided the original work is properly cited.

Copyright (C) the Korean Society of Anesthesiologists, 2021 


\section{CASE REPORT}

Written informed consent was obtained from all patients.

\section{Case 1}

An 89-year-old female was diagnosed with lumbar spinal stenosis with bulging of the L2-L5 intervertebral discs. She had a history of chronic LBP for over 10 years which she took medicine for but had never had operative procedures or interventions. Her medical history included hypertension and hyperlipidemia, for which she was taking aspirin. She was diagnosed with a T12-L3 level compression fracture for which she received L2 level vertebroplasty. She had recently complained of LBP with left sided lumbar radicular pain. She was pharmacologically treated with nonsteroidal antiinflammatory drugs. Her blood tests, such as activated partial thromboplastin time, prothrombin time, and platelet count were all in normal ranges.

After her aspirin was withheld for 5 days, the TFESI was performed under fluoroscopic guidance, with a 22-G Tuohy epidural needle at the left intervertebral foramen between the L4 and L5 levels in the prone position. After penetrating the skin, we checked for the absence of blood in the syringe before advancing the needle. We injected $4 \mathrm{ml}$ of contrast media before drug injection. The contrast was injected and the patient had no particular abnormality and the lateral view image showed that the contrast spread well in the anterior epidural space (Fig. 1). After correctly positioning the needle, lidocaine with $1 \mathrm{mg}$ of dexamethasone in $8 \mathrm{ml}$ total volume was injected. The patient returned home on foot without any particular problem.

The next morning, $24 \mathrm{~h}$ after the procedure, the patient underwent an emergency MRI due to severe lower back pain and complaints of subjective weakness in the left leg. A lumbar spine MRI showed a 14 centimeter epidural hematoma extending from the T11 to the L5 level with cord compression at T11-12-L1-4 (Fig. 2). Physical examination showed that the lower extremity motor function grade was 5/5 and anal sphincter tone was normal. Although large amounts of hematoma were observed, neurological symptoms were believed to not be progressing because epidural bleeding was already clotted and no longer active. However, it is decided that epidural blood aspiration would be performed to relieve the patient's pain by reducing pressure on the nerves. Under fluoroscopy-guided, the epidural space was localized at the L1-2 level with an 18-G Tuohy needle, with the bevel of needle facing cephalad, and using loss of resistance to normal saline in the prone position. After a multi-orifice epidural catheter was inserted $1 \mathrm{~cm}$ into the epidural space, approximately $2.5 \mathrm{ml}$ of blackish-red sticky hematoma was aspirated from the epidural space. High sound pressure aspiration carries risks of causing other conditions, the focus was on reducing pressure in the epidural space by a small amount rather than a large amount. After the aspiration, the patient's back pain decreased significantly. Through consultation with a neurosurgeon, emergency surgery was planned in the event that neurological symptoms occurred. With the patient's consent, she was observed for several days.

The follow-up MRI taken 14 days later showed a decrease in the amount of hematoma and the degree of nerve compression (Fig. 3). The patient was discharged without surgical evacuation and any other symptoms.

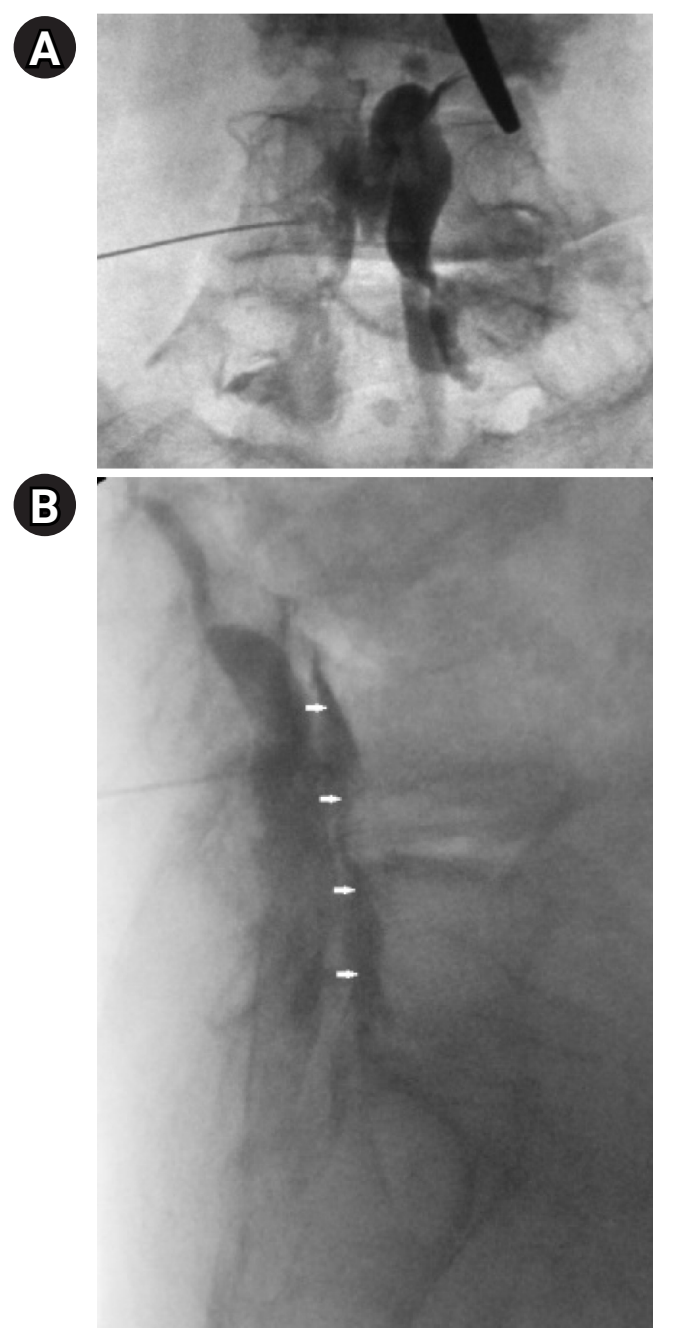

Fig. 1. (A, B) The contrast was injected and spread well in the anterior epidural space (white arrows). 

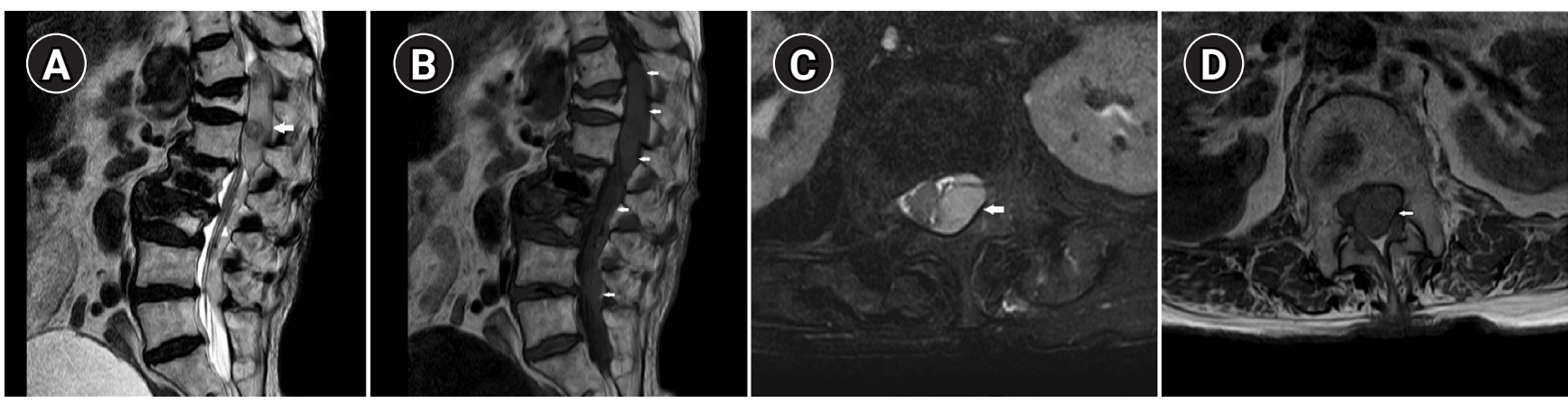

Fig. 2. (A-D) T1- and T2-weighted sagittal and axial MRI images showing the 14-centimeter epidural hematoma (white arrows) at the T11-L4 level. MRI: magnetic resonance imaging.

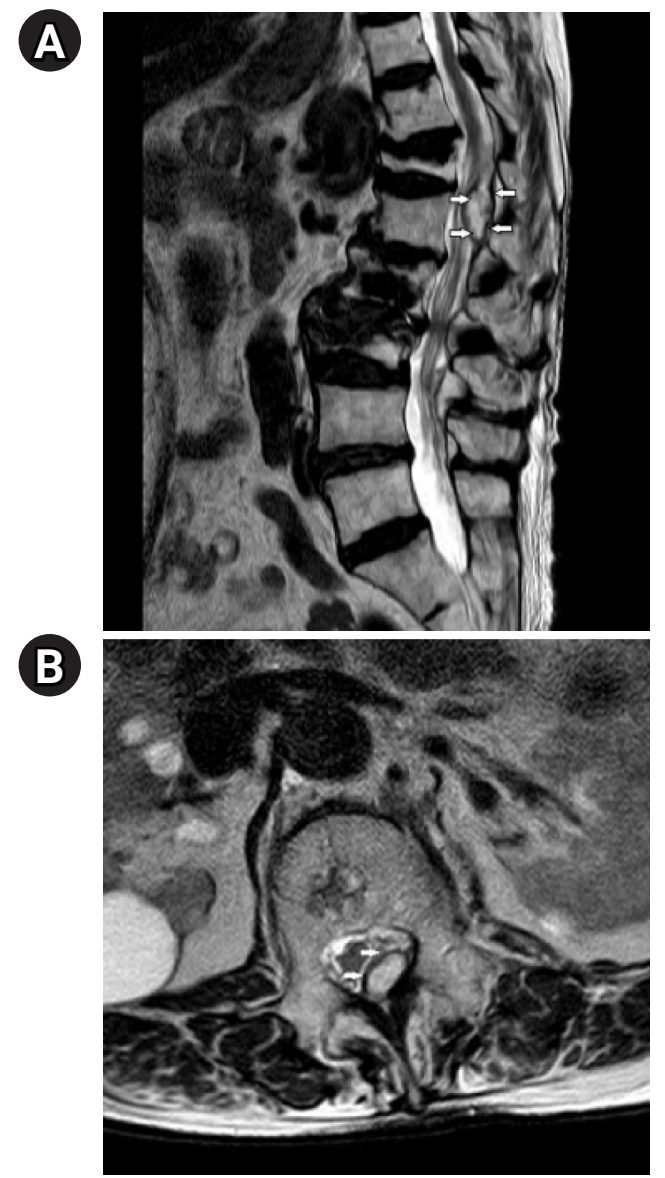

Fig. 3. (A, B) T2-weighted sagittal and axial MRI images showing a decrease in the epidural hematoma (white arrows) and nerve compression. MRI: magnetic resonance imaging.

\section{Case 2}

An 86-year-old female was diagnosed with moderate spinal stenosis in the L2-L5 lumbar spine region. She had a history of chronic LBP and lumbar radicular pain on both sides since 2015. Over the last year, she had been pharmacologically treated with nonsteroidal antiinflammatory drugs and acetaminophen. Her medical history included hypertension and an old cerebrovascular accident, for which she was taking ginko.

She had recently complained of severe back pain and radicular symptoms around the distribution of the L5 nerve root. Neurological examination results were normal with a bilateral motor strength of $5 / 5$ in the lower extremities. Her blood tests were all in normal ranges. She agreed to stop taking ginko during her visit for treatment. She laid prone and a pillow was placed under her lower abdomen. The TFESI was performed under fluoroscopic guidance, with a 22-G Tuohy epidural needle at both intervertebral foramen between the L5 and S1 levels. After penetrating the skin, there was no evidence of intravascular uptake or intrathecal distribution. We injected $3 \mathrm{ml}$ of contrast on both sides, but the anterior spreading did not work well, so we decided to inject another contrast to check anterior spreading. As soon as the contrast was injected to the left, she complained of extreme pain and the fluoroscopy image showed the contrast spreading well. The lateral view showed the contrast to be anterior spreading (Fig. 4).

During recovery, she continuously complained of pain and an inability to move. For pain relief, $25 \mathrm{mg}$ of intravenous pethidine was administered, but the pain did not improve. An MRI scan was performed within three hours which revealed a large amount of epidural and subdural hematoma at the lower T-L spines and sacrum with cord compression at the $\mathrm{T}$ spine (Fig. 5).

Physical examination showed that lower extremity motor function was a grade $5 / 5$ and anal sphincter tone was normal. Under fluoroscopy-guided, the procedure was performed with the patient in a prone position with an 18-G Tuohy needle inserted at the T12-L1 and L3-L4 levels (Fig. 6). After a 20-G multi-orifice epidural catheter was inserted 1 $\mathrm{cm}$ upward into the epidural space, approximately $5 \mathrm{ml}$ of 
diluted watery blood was aspirated from the epidural space. This aspiration occurred three hours after the procedure was performed, so the aspirated blood was significantly more diluted than that of the patient in the first case, and the resistance was not severe. During the procedure, the patient did not complain of any discomfort and her pain decreased significantly after the procedure.
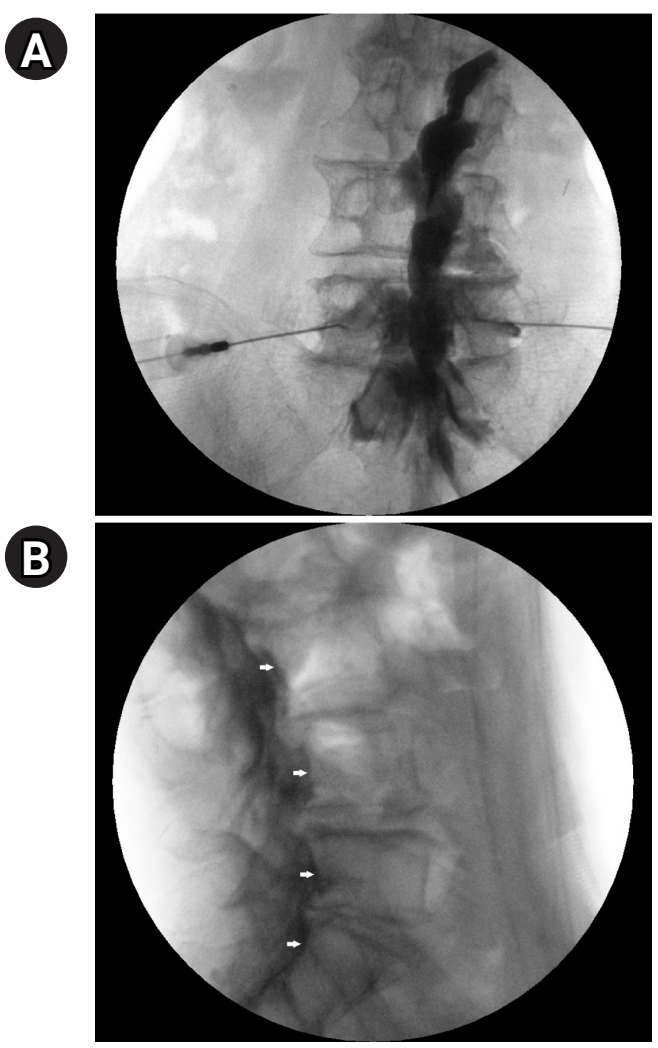

Fig. 4. (A, B) AP/lat. fluoroscopy image showing the anterior epidural space spreading (white arrows).
Emergency surgery was planned in the event that neurological symptoms occurred, but within three days, the patient was able to start walking with reduced pain. A follow-up MRI taken nine days later, the hematoma was shown to be significantly reduced and the patient no longer complained of back pain (Fig. 7). She was discharged from the hospital without any particular complications.
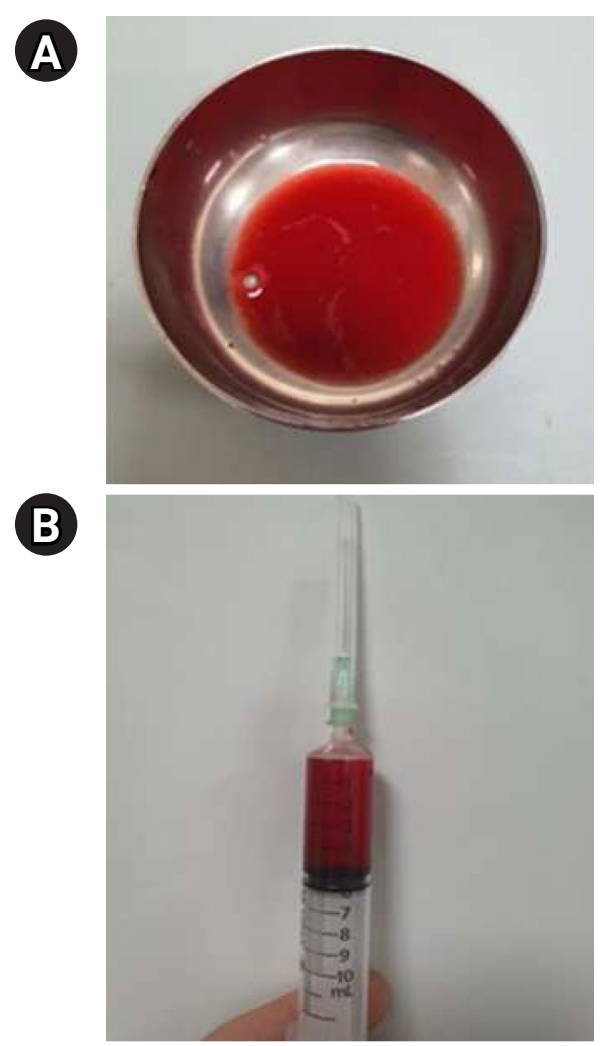

Fig. 6. (A, B) A total of $5 \mathrm{ml}$ of diluted blood aspirated through an epidural catheter.
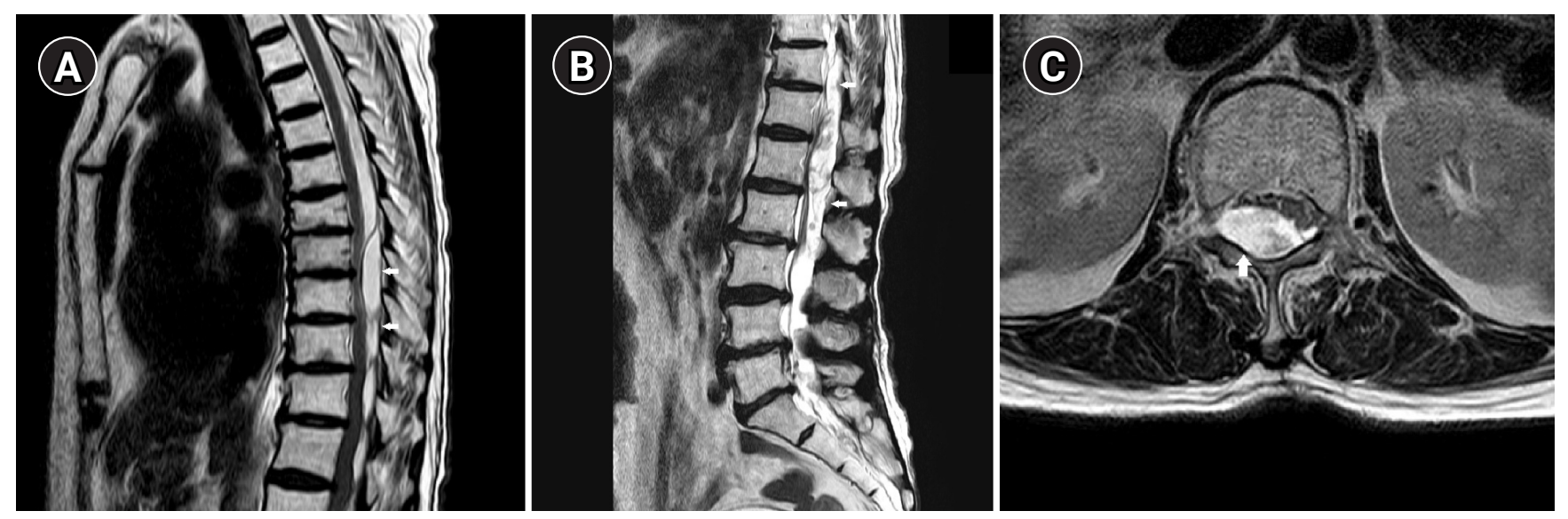

Fig. 5. (A, B) T2-weighted sagittal MRI image showing a large amount of epidural hematoma (white arrows) at the lower T-L spine and clumping of cauda equina at the L1-2 level. (C) T2-weighted axial MRI image at the L1 level showing epidural hematoma and nerve compression (white arrow). MRI: magnetic resonance imaging. 


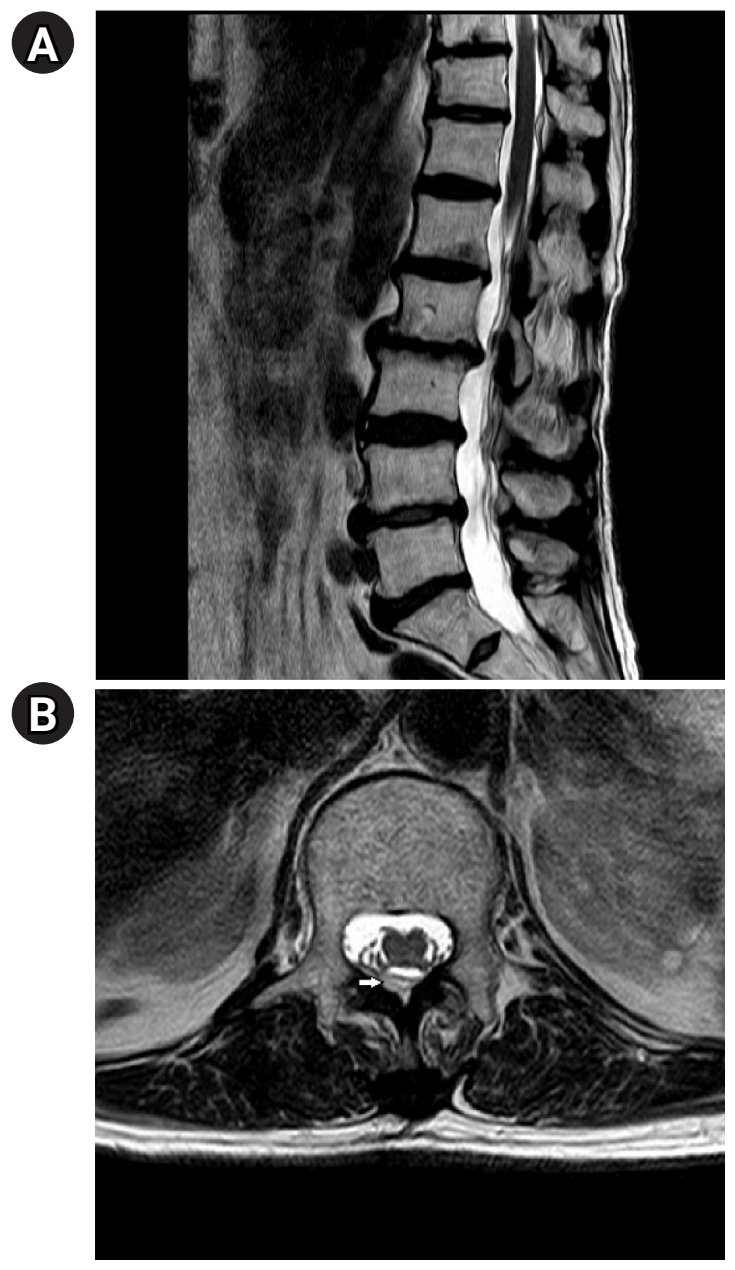

Fig. 7. (A, B) T2-weighted sagittal and axial MRI images showing decreased epidural hematoma (white arrow) and nerve compression. MRI: magnetic resonance imaging.

\section{DISCUSSION}

LBP, which is the most common health problem, causes pain and radiculopathy as a result of nerve irritation and inflammation. Many studies have shown that injecting the appropriate drugs through the epidural space can help relieve radiculopathy pain [2]. This procedure has very rarely caused life-threatening complications, including increased neurological deterioration, intravascular injections, cerebrospinal fluid fistulas, persistent positional headaches, arachnoiditis, hydrocephalus, air embolisms, urinary retention, allergic reactions, stroke, blindness, hematomas, and seizures [3].

The three main techniques for epidural steroid injection in the lumbar spine include transforaminal, interlaminar, and caudal approaches [4]. TFESI is considered to be the most target-specific modality requiring the smallest volume to reach the primary site of pathology. It has been evaluated in an observational study to be a reasonable treatment of lumbar spinal stenosis and can be an alternative to surgery $[5,6]$. The traditional needle target for transforaminal injection is caudad to the inferior margin of the pedicle, which is superior, lateral, and anterior to the targeted exiting nerve. This approach, usually referred to as the safe triangle approach, can be performed with minimal risk of nerve injury, intrathecal puncture, or vascular injection. However, recently, there have been reported cases of paraplegia as a result of the safe triangle approach due to the location of the radicular or radiculomedullary artery in the anterosuperior portion of the foramen [4]. Therefore, significant care needs to be taken even when using the safe triangle approach.

Spinal epidural hematoma (SEH) is a rare condition in which blood accumulates in the epidural space and mechanically compresses the spinal cord. If it improperly managed, it can cause permanent neurologic deficits [7]. Although it is unknown how frequently epidural hematoma occurs as a result of epidural steroid injections, there are surprisingly few case reports of SEH after epidural steroid injections. The incidence may be comparable to the risk of epidural hematoma after epidural anesthesia, which is 1 in 150,000-190,000 [7,8]. The pathogenesis of SEH remains unknown. Most hematomas occur spontaneously, with no known cause. However, some spontaneous lesions are related to vascular anomalies, epidural procedures, hypertension, and physical exertion [7]. Some researchers have suggested that SEH may have developed due to bleeding from the sudden stretching and rupturing of the spinal epidural artery. Patients who suffer arterial rupture also often suffer radicular pain and bleeding originating in the root zone. Others researchers have suggested that it is caused by epidural venous plexus which are composed of thin, valve-less vessels. Spinal epidural venous plexus is usually located at the posterior of the dural sac, so progressive motor and sensory damage often appear delayed [9]. Kim et al. [2] reported on a spinal epidural hematoma occurring at a distance from the transforaminal epidural injection site. They suggested the increasing pressure in the epidural space caused the hematoma rather than direct needle injury. Even simple actions, such as coughing and defecations, increases pressure in the epidural space, so epidural steroid injections can cause large increases in the epidural space pressure, especially in elderly patients with spinal stenosis.

A meta-analysis showed that anticoagulation is the sec- 
ond-most common underlying etiology for spontaneous hematoma formation, following idiopathic occurrences with no identifiable cause [8]. Also, spinal and epidural procedures in combination with anticoagulation were the fifth-most common cause of epidural hematoma. Additional risk factors are age, anatomic abnormalities of the spinal cord and vertebral column, needle size, traumatic needle or catheter placement, epidural techniques being used instead of spinal techniques, indwelling epidural catheters during low molecular weight heparin administration $[7,8]$ and increasing pressure in the epidural space [2]. In the cases in this report, epidural hematomas developed despite adequate discontinuation of anticoagulants in both patients. Physicians performing TFESI should always remember that there are many other factors besides anticoagulants that can cause complications.

Prompt evacuation of the hematoma is generally regarded as the first treatment option for symptomatic spinal epidural hematoma [10]. Decompressive laminectomy is the most common and recommended procedure for removal of spinal epidural hematoma. Epidural hematomas with neurological symptoms are most frequently treated with decompressive laminectomy. There are only a few reported cases of treating them with non-surgical epidural aspiration [2,11-13]. In the absence of neurological deficits, nonsurgical management of epidural hematoma in hemophilia teenagers have also been reported [14]. Surgical treatment of epidural hematoma is pursued depending on whether neurological deficits have occurred. Therefore, it is necessary to closely monitor the occurrence of neurological symptoms. We presented two cases of large amounts of hematoma causing severe back pain that responded to nonsurgical management. In our cases, although there was a large amount of epidural hematoma, there were no neurological symptoms. Neurosurgery was prepared for, but the hematoma was treated by epidural hematoma aspiration.

These reports are believed to be the first of treating epidural hematoma occurring after transforaminal epidural steroid injection through non-surgical hematoma aspiration. If large amounts of epidural hematoma do not show neurological deficits, epidural hematoma aspiration can be considered until the hematoma is absorbed.

\section{CONFLICTS OF INTEREST}

No potential conflict of interest relevant to this article was reported.

\section{DATA AVAILABILITY STATEMENT}

The datasets analysed during the present case are available from the corresponding author on reasonable request.

\section{AUTHOR CONTRIBUTIONS}

Conceptualization: Sung Jun Hong, Sang Soo Kang. Data curation: Go Eun Kim. Formal analysis: Go Eun Kim, Sung Jun Hong. Visualization: Sang Soo Kang. Writing - original draft: Go Eun Kim. Writing - review \& editing: Sang Soo Kang. Resources: Ho Joon Ki, Jae Hyun Park. Software: Ho Joon Ki, Jae Hyun Park. Supervision: Go Eun Kim, Sung Jun Hong, Sang Soo Kang.

\section{ORCID}

Go Eun Kim, https://orcid.org/0000-0002-7223-6317

Sung Jun Hong, https://orcid.org/0000-0002-2466-718X

Sang Soo Kang, https://orcid.org/0000-0002-9347-4883

Ho Joon Ki, https://orcid.org/0000-0002-6643-0391

Jae Hyun Park, https://orcid.org/0000-0002-5276-6994

\section{REFERENCES}

1. Wong AY, Karppinen J, Samartzis D. Low back pain in older adults: risk factors, management options and future directions. Scoliosis Spinal Disord 2017; 12: 14.

2. Kim SI, Lee DH, Kim SH, Cho YH. Spinal epidural hematoma occurring at a distance from the transforaminal epidural injection site: a case report. Medicine (Baltimore) 2019; 98: e16654.

3. Epstein NE. The risks of epidural and transforaminal steroid injections in the spine: commentary and a comprehensive review of the literature. Surg Neurol Int 2013; 4(Suppl 2): S74-93.

4. Mandell JC, Czuczman GJ, Gaviola GC, Ghazikhanian V, Cho $\mathrm{CH}$. The lumbar neural foramen and transforaminal epidural steroid injections: an anatomic review with key safety considerations in planning the percutaneous approach. AJR Am J Roentgenol 2017; 209: W26-35.

5. Davis N, Hourigan P, Clarke A. Transforaminal epidural steroid injection in lumbar spinal stenosis: an observational study with two-year follow-up. Br J Neurosurg 2017; 31: 205-8.

6. Manchikanti L, Singh V, Pampati V, Falco FJ, Hirsch JA. Comparison of the efficacy of caudal, interlaminar, and transforaminal epidural injections in managing lumbar disc herniation: is one method superior to the other? Korean J Pain 2015; 28: 1121. 
7. Al-Mutair A, Bednar DA. Spinal epidural hematoma. J Am Acad Orthop Surg 2010; 18: 494-502.

8. Xu R, Bydon M, Gokaslan ZL, Wolinsky JP, Witham TF, Bydon A. Epidural steroid injection resulting in epidural hematoma in a patient despite strict adherence to anticoagulation guidelines. J Neurosurg Spine 2009; 11: 358-64.

9. Lefranc F, David P, Brotchi J, De Witte O. Traumatic epidural hematoma of the cervical spine: magnetic resonance imaging diagnosis and spontaneous resolution: case report. Neurosurgery 1999; 44: 408-10.

10. Jang JW, Lee JK, Seo BR, Kim JH, Kim SH. Spontaneous resolution of a traumatic cervicothoracic epidural hematoma presenting with transient paraplegia: a case report. Spine (Phila Pa 1976) 2010; 35: E564-7.
11. Gungor S, Aiyer R. Epidural hematoma development contralateral to dura after lumbar transforaminal epidural steroid injection. Pain Manag 2017; 7: 367-75.

12. Choi JJ, Chang YJ, Jung WS, Lee KC, Kim JH, Jo YY. Discordant lumbar epidural hematoma after caudal steroid injection: a case report (CARE-compliant). Medicine (Baltimore) 2017; 96: e7127.

13. Shanthanna H, Park J. Acute epidural haematoma following epidural steroid injection in a patient with spinal stenosis. Anaesthesia 2011; 66: 837-9.

14. Narawong D, Gibbons VP, McLaughlin JR, Bouhasin JD, Kotagal S. Conservative management of spinal epidural hematoma in hemophilia. Pediatr Neurol 1988; 4: 169-71. 